SOME BASIC PROBLEMS OF THE MATHEMATICAL THEORY OF ELASTICITY 


\title{
SOME BASIC PROBLEMS OF THE MATHEMATICAL THEORY OF ELASTICITY
}

\author{
FUNDAMENTAL EQUATIONS \\ PLANE THEORY OF ELASTICITY \\ TORSION AND BENDING
}

BY

\begin{abstract}
N. I. MUSKHELISHVILI
FOURTH, CORRECTED AND AUGMENTED EDITION

MOSCOW 1954

TRANSLATED FROM THE RUSSIAN

$\mathrm{B} \mathrm{Y}$
\end{abstract}

J. R. M. RADOK

SPRINGER-SCIENCE+BUSINESS MEDIA, B.V. 
() 1977 Springer Science+Business Media Dordrecht

Originally published by Noordhoff International Publishing in 1977

All rights reserved. No part of this publication may be reproduced, stored in a retrieval system, or transmitted, in any form or by any means, electronic, mechanical, photocopying, recording or otherwise without the prior permission of the copyright owner.

ISBN 978-90-481-8245-9 ISBN 978-94-017-3034-1 (eBook)

DOI 10.1007/978-94-017-3034-1

Reprint of the second English edition 


\section{AUTHOR'S FOREWORD TO THE SECOND ENGLISH EDITION}

I was pleased to learn that the need has arisen for a second edition of my book, which will be based on the fourth Russian edition of 1954 .

It is for me a plesant opportunity to express my deep gratitude to Dr. J. R. M. RADOK, on whose initiative the English edition of this book has been published, for his beautiful translation which considerably assisted in the spreading of the methods and results studied in this book beyond the boundaries of the USSR.

I likewise wish to thank Professor I. S. Sokolnikoff of Los Angeles, U.S.A. who even before the publication of the English edition of my book studied a number of these results in his widely known course of the theory of elasticity which also greatly helped foreign readers to come into contact with this work.

Finally, I wish to express my heartfelt thanks to the publishing house of $\mathrm{P}$. NOORDHOFF for the magnificent appearance of the book.

Tbilisi, March 1962.

N. MUSKHelishvili 


\section{FOREWORD TO THE FIRST ENGLISH EDITION.}

The appearance of an English translation of Muskhelishvili's prizewinning monograph on the mathematical theory of elasticity is certain to be greeted with enthusiasm by Western European scholars. Although a version of this monograph was first published in Russian some twenty years ago, the general methods of solution of two-dimensional elastostatic problems developed by Muskhelishvili and his co-workers still remain relatively unknown outside Russia.

Mr. J. R. M. Radok and his Publishers, P. Noordhoff of Groningen, have performed an extremely valuable service by making available to Western scholars a translation of one of the most significant contributions to the theory of elasticity since the publication of Love's celebrated Treatise on the Mathematical Theory of Elasticity.

Los Angeles, California, 1952.

I. S. SOKOLNIKOFF 


\section{TRANSLATOR'S PREFACE TO THE FIRST ENGLISH EDITION.}

In preparing this translation, I have taken the liberty of including footnotes in the main text or inserting them in small type at the appropriate places. I have also corrected minor misprints without special mention. The Chapters and Sections of the original text have been called Parts and Chapters respectively, where the latter have been numbered consecutively. The subject index was not contained in the Russian original and the authors' index represents an extension of the original list of references. In this way the reader should be able to find quickly the pages on which any one reference is discussed. The transliteration problem has been overcome by printing the names of Russian authors and journals also in Russian type.

While preparing this translation in the first place for my own information, the knowledge that it would also become accessible to a large circle of readers has made the effort doubly worthwhile. I feel sure that the reader will share with me in my admiration for the simplicity and lucidity of presentation.

I should like to thank Professor E. STERNBERG of Illinois Institute of Technology at Chicago for loaning me his copy of Muskhelishvili's book through the good offices of Professor F. S. SHAw of the Brooklyn Polytechnic Institute, New York, Professor W. S. Hemp of the College of Aeronautics, Cranfield, England, for reading my translation in manuscript and Professor I. S. SokolnIKOFF of the University of California for reading the second proof and for fulfilling my wish by writing his foreword to the book. Finally, I must express my gratitude to the publishers for making the printing of this book possible and for their constant willingness to meet all special requests.

Zürich, November 1952.

J. R. M. RADOK 


\section{EXTRACT FROM THE AUTHOR'S PREFACE TO THE FIRST RUSSIAN EDITION.}

This book reproduces, in a considerably revised and enlarged form, the contents of a course of lectures, delivered by me in Spring 1931 at the invitation of the Seismological Institute of the Academy of Sciences of the U.S.S.R. before the scientific workers of the Institute, and of lectures delivered in 1932 before post-graduate students of the PhysicoMathematical Institute of Mathematics and Mechanics at the University of Leningrad. The lectures were intended for persons acquainted with the principles of the theory of elasticity and were to be devoted to separate fundamental questions the choice of which was largely left to me; I naturally dwelt on subject matter in which I had been working myself.

Thus, this book deals only with a few chapters of the theory of elasticity each of which receives fairly complete treatment. I shall not touch here on the subject matter of the book an idea of which may be gained from the list of contents, but I consider it necessary to make the following comments.

Seeing that the problems considered in this book may prove of interest to a wider circle of people, in particular to those whose work requires application of the theory of elasticity, I have tried to make the exposition as far as possible intelligible for readers who are only familiar with the fundamentals of differential and integral calculus and the elementary theory of functions of a complex variable. Thus, for example, problems involving integral equations are relegated to separate sections which may be passed over without impairing the understanding of the remainder; Part I which deals with the foundations of the mathematical theory of elasticity (it contains even more than is required) is intended for readers not specializing in the theory of elasticity. In order to make the text more accessible, I refrained from employing tensor calculus which I used in my lectures at the Seismological Institute; an elementary introduction to tensors is given in Appendix 1. Appendices 2 and 3 are devoted to some aspects of elementary mathematics which are necessary for the understanding of the subject matter of the book and which, as a rule, are insufficiently elucidated in elementary courses on analysis.

Leningrad, Spring 1933.

N. MUSkHeLISHVILI 


\section{PREFACE TO THE THIRD RUSSIAN EDITION.}

The second edition of this book, which was published in 1935 almost immediately after the first (which appeared in 1933), has been out of print for a long time, but, as I was engaged on other work, I have only now been able to start the preparation of a new edition. The warm reception given to my book and the high distinction with which it was favoured made it imperative to treat its reissue with special attention. To this was added the circumstance, highly gratifying to me, that soon after the appearance of the first editions many papers were published in which the methods expounded by me were applied to different concrete problems and also substantially amplified and generalized. It is natural that the new edition should at least reflect the main results of these papers as well as some results obtained by me. I have tried to accomplish this, but I am afraid that some papers may still have escaped my notice for which omission I tender my apologies to their authors.

The general design of exposition in this edition has remained the same as before. However, the text of the book, with the exception of the first two and last Parts *, has been thoroughly rewritten and considerably enlarged. Two new Parts have been added, namely the fourth and the sixth. The contents of the fourth Part are only to a negligible extent taken from the preceding edition; in Part VI, results are given which have been obtained by me and also by other authors since the publication of the preceding edition, $* *$ if no account is taken of the few problems whose solution had been given in the previous edition, but by means of other methods.

* However, Chapter 25 has been considerably enlarged and in this way the theory of extension and flexure of compound bars has attained an aspect of completeness.

** A large part of these results were introduced by me into my book "Singular Integral Equations" (Moscow-Leningrad 1946), but now I find that their natural place is in the present book. They will accordingly be omitted from the following edition of "Singular Integral Equations". Here, the exposition of these results has been rearranged in order to make it independent of the above-mentioned book. 
Although I do not think it possible or necessary to indicate all the changes and additions in the text of the earlier editions, I must draw the reader's attention to the new arrangement of Chapters 14-16 (Chapter 17 is new). Compared with the previous editions, the results contained in these chapters are not new, but the method by which they have been obtained has been replaced by a different one which seems to me to be more germane to the matter under consideration. However, I should like to mention that the new method (which was influenced by the work of $\mathrm{J}$. Plemelj on the theory of functions of a complex variable, published long before the first edition of my book, but unfortunately unknown to me at the time) leads to the same calculations as the former method. For this reason and also because of the, say, greater complexity of the new method, I am not certain that I acted rightly in introducing this change. Be that as it may, a comparison of the new and the old approach may prove useful.

In conclusion, I should like to add that as far as possible I have carefully quoted the authors of any results which I have used, just as I have done with regard to some of my own results, at times even of minor importance and adduced merely as examples. I have followed this practice, not because I attach exaggerated importance to these results, but only to avoid puzzling the reader who might not be acquainted with the previous editions of my book and who might have encountered material taken from it without clear indication of its original source in some other publications (mostly non-Russian).

To simplify reference, the quoted works have been listed at the end of the book in alphabetical order. In references, the author is named and the number of his publication, according to this list, is given in square brackets. The first edition was greatly assisted to its success by the preface of the late Academician Alexei Nikolaevich Krylov whose outstanding scientific and public merits are well known to all and for whom I shall always entertain feelings of profound gratitude and respect. Krylov's preface is reproduced below without any changes. I have not been able to fulfil in this edition the wish expressed by Krylov at the end of his preface concerning the development of numerical methods of solution. While realizing the importance of his request, I felt that I would be unable to meet it sufficiently well. Nevertheless, Krylov's wish has been fulfilled by other authors, referred to in the text of the book. 


\section{PREFACE TO THE FOURTH RUSSIAN EDITION.}

This edition differs from the preceding one by numerous additions and changes in the text most of which are small, but at times rather essential. In this respect the author had in mind the reader concerned mainly with practical applications as well as the reader interested in the mathematical aspects of the subject.

I wish to use this opportunity to express my sincere gratitude to G. F. MANDJAVIDZE who read with great interest the proofs of the entire book, noted a number of slips and made several critical comments which led to improvements of the study.

I also wish to thank A. IA. GoRgIDze who read the proofs of the first and seventh chapters and performed certain computations.

Tbilisi, December 1953.

N. MUKSHelishvili 


\section{PREFACE OF ACADEMICIAN A. N. KRYLOV TO THE FIRST EDITION *}

Elasticity is a fundamental property of all substances occurring.in Nature. This property must be ascribed even to that imaginary ether whose very existence is now acknowledged, now disputed by Physics, and which at the same time is so widely used in practice.

Thus, it may be said that the elasticity of bodies has been unwittingly used since prehistoric times for all kinds of buildings from the huts of savages to the Coliseum and majestic palaces and temples, for all tools and instruments of everyday life and for all kinds of weapons. As primeval man or a contemporary Papuan would use the elasticity of his material when making a bow for his flint or bone-headed arrow, so Vickers \& Armstrong rely on the very same property for their 150-ton-16-inch guns for the battleships Nelson and Rodney or for the fortifications of Dover. The only difference is that the Papuan makes his bow by methods arrived at over countless generations as if by natural selection whereas Vickers \& Armstrong design their guns by methods based on mathematical calculations developed by our own Academician Gadolin only 60 years ago. If therefore man's utilization of the property of elasticity has gone on from time immemorial, yet the first attempt at its scientific foundations only dates back 295 years and it was undertaken by Galilei who described it in his famous Discourses, published in 1638.

Forty years later, the Englishman Hooke gave the solution to his anagram "ceiiinosssttuv": ut tensio sic vis, which contained the fundamental law of the elasticity of materials.

After another 60 years, the works of Daniel Bernoulli and Euler appeared in the Transactions of the St. Petersburg Academy of Sciences, which gave the equations still used in most practical calculations.

Yet 80 years later, the French engineer Navier adopted quite a different approach to the study of the elasticity of matter by giving its general theory and setting up the equations of equilibrium and of motion. This theory was further developed by the famous mathematicians Cauchy and Poisson who applied it first to the study of the propagation of waves

* The preface by Krylov has been reprinted without alterations from the first edition. Since Krylov analyses the contents of the book Part by Part, it should be noted that Part III of the first edition, considerably enlarged, constitutes Part V, while Part IV of the first edition corresponds to Part VII of the present edition. 
in elastic media, i.e., of sound, since they did not admit at that time the undulatory theory of light; in his course of mechanics which Poisson gave at the Ecole Polytechnique he developed the theory of Bernoulli and Euler.

At the same time, at the Institute of Communication Engineers founded a few years earlier at St. Petersburg, there were two French engineers, Lamé and Clapeyron, who as professors held the rank of lieutenant-colonel in the Russian Army. They turned towards developing Navier's theory for the purpose of applying it to building and construction; however, soon after his accession, Nicholas I dismissed them from the Russian service and sent them home.

Here, in 1852, Lamé also published the first treatise on the theory of elasticity under the title "Leçons sur la théorie mathématique de l'élasticité des corps solides" which has become a classic and has not lost its significance even today.

Together with the development of railway construction, of steel railway bridges, of huge buildings, with the development of machine construction and shipbuilding, with the setting up of laboratories for the scientific investigation of the elastic properties of materials and for their testing on qualities stipulated by contract went the rapid development of the study of the elastic properties of materials and their proper utilization in building practice.

This development followed two trends: on the one hand, the mathematical theory of elasticitity was worked out in the form in which it had been created by Navier and first set out by Lamé, on the other hand, a simplified theory was developed which was given the name of the Theory of Strength of Materials and which was based on methods, the foundations of which were laid by Bernoulli and Euler.

It is remarkable that the formulae and conclusions of Lamé had their first practical application on the initiative of Gadolin in the project of steel guns reinforced by rings which were first produced by Krupp. It was only then that the Obukhovsky plant was founded. Frequently, practice itself has required the examination of the conclusions of the simplified theory by the more severe and exact theory, so that it might approximate more closely to reality and to ensure that the simplifications did not lead too far away from the truth and that, on their account, the very point of the matter was not disregarded.

In many cases, research has been stimulated by notable collapses of gigantic railway bridges, correctly constructed, one would have thought, 
and calculated according to all the rules and norms, or by cracks and rents in the deck plates of ocean steamers or by cases of loss of fast liners which, after collision with small vessels, broke in two and sank. In the last case, facts about the fractures could be clearly judged by the different inclinations of the masts before they finally submerged.

The theory of elasticity would often help and give an exact quantitative explanation of the causes of the accident, and once the causes were known it was not difficult to find the means to prevent the harmful consequences.

About thirty years ago reinforced concrete began to be used for building purposes; this new application rapidly developed and has now attained vast importance. At that juncture, new problems arose and for the solution of many of them the simplified methods of Bernoulli and Euler were ineffective, so that the theory of elasticity found a number of new applications.

Hence it is clear how important the study of this subject is at present with our tremendous building activity in its countless different forms, and therefore N. I. Muskhelishvili's book fills an essential need.

In his preface, the author refers the reader, wishing to acquaint himself with the subject matter of the book, to its list of contents, but the dry list gives an insufficient idea not only of the method of exposition, but even of its very contents. I shall therefore try to give a brief introduction to the nature of the exposition, noting, above all, its originality, thanks to which a conciseness has been obtained which enabled the author to present in his small book such vast, and to a great extent, new material and at the same time to preserve full clarity of treatment.

Professor Muskhelishyilt's BoOK Contains four Parts:

Part I. Fundamental equations of the mechanics of elastic bodies.

Here, in 75 pages, all the general principles of the theory of elasticity are set out, viz., a) Analysis of stress, b) Analysis of strain, c) Relation between stress and strain, $d$ ) The equilibrium equations of an elastic body and the two fundamental problems: 1) to determine the state of stress of a body when the forces acting on it are given, and 2) to determine the state of stress of a body when the displacements of points of the boundary of the body are given. 
Part II Plane Problem. General formula e and elementary applications

These 100 pages deal with the plane problem and show the main methods for its solution. The solution is achieved by the help of the stress function and its complex representation; first, an account of the general theory of methods is given, and then the methods are practically developed in a number of examples. Of these examples we shall note a) tension of a plate weakened by a circular hole, $b$ ) effect of a concentrated force applied at a point of an unbounded plane, $c$ ) effect of a concentrated couple, $d$ ) examination of the state of stress in a ring under the action of given forces, $e$ ) flexure of a circular beam, $f$ ) general theory of thermal strains and stresses.

Part III Applieation of conformal mapping and complex integration to the plane problem

These 108 pages contain the theory and examples of conformal mapping and of its application to the transformation of equations of the plane problem and of its boundary conditions. After that, the general method of solution of the fundamental problems is developed and the solution of these problems is illustrated by the example of the continuous ellipse. Next, the author develops the theory of Cauchy integrals and gives the generalized formulae which he employs further on.

Having derived the general solution of the fundamental problems for regions bounded by one contour and having reduced this solution to that of Fredholm equations, the author gives the solution of many examples for regions of different form; in particular, he examines the solution of the fundamental problems for the plane with elliptic perforations and with nuclei; after that, he gives the solution of both fundamental problems for the half-plane and for regions of a more general character.

All these solutions are obtained, not by fortuitous particular methods, but by the application of a common method based on properties of Cauchy integrals discovered by the author.

When reading this chapter, I inadvertently remembered the Spring meeting of the Society of Shipbuilding Engineers in 1898. At this meeting, Professor Hele-Shaw showed, for the first time, the equipment by which he projected on a screen, with marvellous distinctness, the flow of a liquid and showed how the streamlines bypassed different kinds of obstacles. In the following year, I was again in London at the Spring meeting of the 
Society. Among other papers, the Norwegian engineer Bruhn read a paper on the influence of perforations and apertures in the deck on the overall strength of vessels, and since, not long before, the huge steamer "City of Rome" broke up after a collision with a sailing ship a few miles off New York, and since this catastrophe was still fresh in everybody's mind, Bruhn's paper was received with special interest. To study this influence, he took an oblong sheet of rubber, ruled it with straight lines running parallel and perpendicular to its long side thus forming squares and made perforations in it of various forms; having stretched the sheet lengthwise, he traced the form of the curves into which the originally straight lines on the sheet had been transformed. From these lines he obtained a picture of the strain distribution, and consequently of the stress distribution. By use of similar models, Bruhn proposed to study the solutions of the plane problems which N. I. Muskhelishvili has solved so brilliantly analytically.

Accidentally, one of Bruhn's perforations had the same shape as one of the obstacles in Hele-Shaw's experiments, demonstrated the year before. There was almost complete identity between Bruhn's curves and HeleShaw's streamlines. Since nobody drew attention to this fact, I obtained the Reports of the Society for the preceding year, requested leave to be heard and explained that the coincidence was by no means accidental, that Bruhn's method was the mechanical and Hele-Shaw's method the hydrodynamical solution of the same generalized problem of Dirichlet and that there was no need for Bruhn's complicated models and measurements nor for the tracing of the curves obtained; in fact, one had only to insert an obstacle of a corresponding form into Hele-Shaw's apparatus and to take a photograph by projecting them on ordinary sensitive paper, in order to obtain a map of the strain distribution. Such a juxtaposition of phenomena from quite different fields came altogether unexpectedly to the meeting and its old chairman, the highly experienced engineer Benjamin Martel, made special acknowledgement of my remarks in his final address.

The flow of gases and liquids is now studied by many methods which are modifications of that of Hele-Shaw as well as of others, because of its considerable significance in aerodynamics. It might possibly be a worthwhile task to compare such an "aerodynamic spectrum" with the solutions given by N. I. Muskhelishvili, and hence to elaborate an experimental method for solving the plane problems of the theory of elasticity.

Another method which has been so splendidly developed by Academician N. N. Pavlovsky happens to come to my mind, namely the electri- 
cal solution of the hydrodynamic problem of determining equipotential and stream lines on a conducting plate with perforations of given shape.

All this represents a realisation of conformal transformations, so elegantly developed in analytic form by Muskhelishvili, and shows the interrelationship by way of common differential equations between apparently quite disconnected branches of physics.

In this context, one might usefully remember that the stress function itself was introduced into the theory of elasticity by the famous Astronomer Royal Sir James Biddel Airy who, I believe, was director of Greenwich Observatory for more than 50 years. At the beginning of the 1860's, he built for the Observatory a new large meridian line with a telescope having an 8-inch object lens. He had to count with the flexure of the telescope under the weight of the lens and ocular and of other devices, a fact which had caused errors up to 2 arc seconds at the Paris Observatory, errors which are inadmissible in such accurate observations with significant measurements in decimal seconds.

Part. IV Torsion and flexure of homogeneous and compound bars

This Part provides an excellent account of Saint-Venant's problem and also studies compound bars, so important for reinforced concrete construction, by entirely new methods developed by the author.

Even at a cursory reading of the book, the originality of the solutions is evident not only as far as the quite new problems proposed by the author are concerned, but also with regard to problems that have long ago been solved by other authors. Thus, in Part I, which by the nature of its contents deals with well established results, the author elucidates many questions more fully and distinctly than has been done before; for example, the new deduction of the compatibility conditions of Saint-Venant and Beltrami-Michell belongs to the author. In Part II, the author offers a new strict deduction of G. V. Kolosov's formulae and of a number of other formulae; the detailed study of the analytic character of the solutions of the plane problem for multiply connected and infinite regions enables us to discover fallacious conclusions committed by other investigators.

Also of significance is the author's general investigation of the effect of concentrated forces and the presentation of their most general ex- 
pressions; this again helped to reveal mistakes in other papers. The establishment of the connection between thermal stresses and multi-valued displacements also belongs to Muskhelishvili.

All examples in this Part have either been solved by the author for the first time or, if solved before, more complicated methods had been applied for their solution.

Part III belongs entirely to the author in the originality and in the generality of the problems solved in it as well as in the originality of the methods applied. How important this method is may be seen from the fact that in $\S 68$ ( $\S 82$ of the 3rd edition) the author gives the general solution of the second fundamental problem for an infinite plate with an elliptic hole on two pages of large print. A particular case of this problem was solved by L. Föppl in the Zeitschrift für angewandte Mathematik und Mechanik, his solution occupying five large pages of small print which, if set up in our academic type, would fill about twenty pages; in $\S 69$ ( $\$ 82 \mathrm{a}$ of the $3 \mathrm{rd}$ edition), an example is solved in a few lines the simplest particular case of which agrees with that of Föppl.

In Part IV, as previously mentioned, all matter relating to nonhomogeneous bodies, beginning with the very statement of the problem, belongs to Muskhelishvili.

From this short sketch may be seen the rich content as well as the variety and importance of the problems covered in this book and the originality and generality of the methods applied for their solution.

There only remains to express the wish that in future editions, which without doubt will be required, the author illustrate the general deductions and formulae by numerical examples, by diagrams and by indications as to the number of ordinates or subdivisions required for approximate integration in order to ensure accuracy within, say, $\frac{1}{2} \%$. He will thereby render a great service to engineers and make his excellent book more accessible to those people who will apply its deductions to the solution of the purely practical problems of the building industry.

Academician A. KRYLOV 


\section{CONTENTS}

PART I - Fundamental equations of the mechanics of an elastic body. 1

Chapter 1. Analysis of stress.

$\S$ 1. Body forces. . . . . . . . . . . . . 5

$\S$ 2. Stress. . . . . . . . . . . . . . . . 6

$\S 3$. Components of stress. Dependence of stress on the orientation of the plane . . . . . . . . . . 7

$\S$ 4. Equations, relating components of stress . . . . . 10

$\S 5$. Transformation of coordinates. Invariant quadratic form. Stress tensor. . . . . . . . . . . . . . 14

$\S$ 6. Stress surface. Principal stresses. . . . . . . . . . 17

$\S 7$. Determination of principal stresses and axes . . . . 22

§ 8. Plane stress... . . . . . . . . . . . 23

Chapter 2. Analysis of strain.

$\S$ 9. General remarks . . . . . . . . . . . . . . 28

$\S$ 10. Affine transformation . . . . . . . . . . . . . . 29

$\S$ 11. Infinitesimal affine transformation. . . . . . . . 31

$\S$ 12. Decomposition of infinitesimal transformations into pure deformation and rigid body motion. . . . . . 32

$\S$ 13. The invariant quadratic form, connected with deformation. The strain surface, principal axes. Transformation of coordinates . . . . . . . . . . 38

$\S$ 14. General deformation .. . . . . . . . . . . . . 41

$\S 15$. Determination of displacements from components of strain. Saint-Venant's condition of compatibility . . 44

Chapter 3. The fundamental laW OF the theory of elasticiTY; THE BASIC EQUATIONS.

$\S 16$. The fundamental law of the theory of elasticity (generalized Hooke's Law) . . . . . . . . . 52

$\S$ 17. Isotropic bodies... . . . . . . . . . 56 
$\S 18$. The basic equations for the statics of an elastic isotropic body. . . . . . . . . . . . . 60

$\S 19$. The simplest cases of elastic equilibrium. The basic elastic constants... . . . . . . . . . . 62

$\S 20$. The fundamental boundary value problems of static elasticity. Uniqueness of solution . . . . . . . . 66

$\S 21$. Basic equations in terms of displacement components 73

$\S$ 22. Equations in terms of stresses . . . . . . . . . . 74

$\S 23$. Remarks on the effective solution of the fundamental problems. Saint-Venant's principle. . . . . . . . 77

$\S$ 24. Dynamic equations. The fundamental problems of the dynamics of an elastic body . . . . . . . . 78

PART II - General formulae of the plane theory of elasticity . . . .

Chapter 4. BASIC EQUATIONS OF THE PLANE THEORY OF ELASTICITY.

$\S$ 25. Plane strain. . . . . . . . . . . . . . . . . .

$\S 26$. Deformation of a thin plate under forces acting in its plane. . . . . . . . . . . . . . . 92

$\S 27$. Basic equations of the plane theory of elasticity . . . 96

$\S 28$. Reduction to the case of absence of body forces . . . 101

Chapter 5. Stress function. Complex Representation of THE GENERAL SOLUTION OF THE EQUATIONS OF THE PLANE THEORY OF ELASTICITY.

$\S$ 29. Stress function . . . . . . . . . . . . . . 105

\$ 30. Determination of displacements from the stress function 107

$\S 31$. Complex representation of biharmonic functions . . 110

$\S$ 32. Complex representation of displacements and stresses 113

$\S 33$. The physical meaning of the function $f$. Expressions for the resultant force and moment . . . . . . . . 116

$\S 34$. Arbitrariness in the definition of the introduced functions . . . . . . . . . . . . . . . 118

$\S 35$. General formulae for finite multiply connected regions 121

$\S 36$. Case of infinite regions. . . . . . . . . . . . . . 126

$\S 37$. Some properties following from the analytic character of the solution. On analytic continuation across a given contour . . . . . . . . . . . 131

$\S$ 38. Transformation of rectilinear coordinates. . . . . 137 
$\S$ 39. Polar coordinates . . . . . . . . . . . . . . . . 140

$\S 40$. The fundamental boundary value problems. Uniqueness of Solution . . . . . . . . . . . . . . . . . 141

$\S 41$. Reduction of the fundamental problems to problems of complex function theory . . . . . . . . . . . . . 147

$\S$ 41a Supplementary remarks . . . . . . . . . . . . . . 156

$\S 42$. Concept of the regular solution. Uniqueness of a regular solution. . . . . . . . . . . . . 158

$\S$ 43. On concentrated forces, applied to the boundary . . . 162

$\S 44$. Dependence of the state of stress on the elastic constants 164

Chapter 6. Multi-valued displacements. Thermal stresses.

$\S$ 45. Multi-valued displacements. Dislocations . . . . . 167

$\S$ 46. Thermal stresses . . . . . . . . . . . . . . . . 170

Chapter 7. Transformation of the basic formulae for CONFORMAL MAPPING.

$\S$ 47. Conformal transformation. . . . . . . . . . . . . 176

$\S 48$. Simple examples of conformal mapping.

$1^{\circ}$. Bilinear function . . . . . . . . . . . 180

$2^{\circ}$. Pascal's limaçon.. . . . . . . . . . . . 185

$3^{\circ}$. Epitrochoids . . . . . . . . . . . . . 186

$4^{\circ}$. Hypotrochoids . . . . . . . . . . . . 187

5․ Elliptic rings. . . . . . . . . . . . . . . . . 188

$\S$ 49. Curvilinear coordinates, connected with conformal transformations into circular regions. . . . . . . 190

$\S 50$. Transformation of the formulae of the plane theory of elasticity . . . . . . . . . . . . 192

$\S 51$. Boundary conditions in the image regions . . . . . 194

PART III - Solution of several problems of the plane theory of elasticity by means of power series . . . . . . . . . . . . . . . . . 197

Chapter 8. ON Fourier series.

$\S 52$. On Fourier series in complex form . . . . . . . . 199

$\S 53$. On the convergence of Fourier series . . . . . . 202

Chapter 9. SOlution FOR REgIONS, BOUNDED by a CIRCle.

$\S 54$. Solution of the first fundamental problem for the circle. 
$\S 55$. Solution of the second fundamental problem for the circle -

$\S 56$. Solution of the first fundamental problem for the infinite plane with a circular hole.

$\S 56 a$. Examples.

$1^{\circ}$. Uni-directional tension of a plate, weakened by a circular hole . . . . . . . . . . . . 211

$2^{\circ}$. Bi-axial tension ............ . 214

$3^{\circ}$. Uniform normal pressure applied to the edge of a circular hole.............. . 214

$4^{\circ}$. A concentrated force, applied at a point of the infinite plane. . . . . . . . . . . 215

$5^{\circ}$. Concentrated couple . . . . . . . . 216

$\S 57$. On the general problem of concentrated forces . . . 217

$\S 58$. Some cases of equilibrium of infinite plates, containing circular discs of different material.

$1^{\circ}$. Infinite plate with a circular hole into which an elastic circular disc with an originally larger radius has been inserted.

222

$2^{\circ}$. Stretching of plates with inserted or attached rigid discs ................ 224

$3^{\circ}$. Stretching of plates with inserted or attached elastic discs

Chapter 10. THE CIRCULAR RING.

$\S 59$. Solution of the first fundamental problem for the circular ring. . . . . . . . . . . . 230

$\S$ 59a. Examples.

$1^{\circ}$. Tube subject to uniform external and internal pressures. . . . . . . . . . . . 235

$2^{\circ}$. Stress distribution in a ring, rotating about its centre .............. 236

$\S 60$. Multi-valued displacements in the case of a circular ring 237

$\S$ 61. Supplement. Bending of a curved beam . . . . . . 242

$\S 62$. Thermal stresses in a hollow circular cylinder . . . . 246 
Chapter 11. Application of CONFORMal mapping.

$\S$ 63. Case of simply connected regions . . . . . . . 250

$\S$ 64. Example of application of mapping on to a circular ring. Solution of the fundamental problems for a continuous ellipse . . . . . . . . . . . . 257

PART IV - On Cauchy integrals . . . . . . . . . . . . . . 265

Chapter 12. Fundamental properties of Cauchy integrals.

$\S 65$. Notation and terminology . . . . . . . . 267

$\S 66$. Cauchy integrals. . . . . . . . . . . . . 270

$\S 67$. Values of Cauchy integrals on the path of integration. Principal value . . . . . . . . . . . 271

$\S 68$. Boundary values of Cauchy integrals. The Plemelj formulae . . . . . . . . . . . . . 276

$\S 69$. The derivatives of Cauchy integrals . . . . . . . . 279

$\S 70$. Some elementary formulae, facilitating the calculation of Cauchy integrals . . . . . . . . . . . . . . 281

$\S 71$. On Cauchy integrals, taken along infinite straight lines

$\S 72$. On Cauchy integrals, taken along infinite straight lines (continued) . . . . . . . . . . . . . . 296

Chapter 13. Boundary VAlUes of holomorphic Functions.

$\S$ 73. Some general propositions . . . . . . . . . 298

$\S$ 74. Generalization . . . . . . . . . . . . 301

$\S$ 75. Harnack's theorem. . . . . . . . . . . . . 301

$\S 76$. Some special formulae for the circle and the half-plane 303

$\S 77$. Simple applications: solutions of the fundamental problems of potential theory for a circle and half-plane 308

PART V - Application of Cauchy integrals to the solution of boundary problems of plane elasticity . . . . . . . . . . . . 315

Chapter 14. General solution of the fundamental proBLEMS FOR REGIONS BOUNDED BY ONE CONTOUR.

$\S 78$. Reduction of the fundamental problems to functional equations . . . . . . . . . . . . . . 317

$\S 79$. Reduction to Fredholm equations. Existence theorems 323

$\S 79$ a. On some other applications of the preceding integral equations . . . . . . . . . . . . 
Chapter 15. Solution of the fundamental problems for REGIONS MAPPED ON TO A CIRCLE BY RATIONAL FUNCTIONS. EXTENSION TO APPROXIMATE SOLUTION FOR REGIONS OF GENERAI, SHAPE.

$\S 80$. Solution of the first fundamental problem for the circle 334 $\S 80$ a. Examples.

$1^{\circ}$. Circular disc under concentrated forces, applied to its boundary. . . . . . . . . . . . . 338

$2^{\circ}$. Disc under concentrated forces and couples acting at internal points. . . . . . . . . . . . . 342

$3^{\circ}$. Rotating disc with attached discrete masses . . 345

$\S 81$. Solution of the second fundamental problem for the circle . . . . . . . . . . . . . . 346

$\S$ 82. Solution of the first fundamental problem for the infinite plane with an elliptic hole

$\S$ 82a. Examples.

$1^{\circ}$. Stretching of a plate with an elliptic hole .. .

$2^{\circ}$. Elliptic hole the edge of which is subject to uniform pressure . . . . . . . . . . . . 353

$3^{\circ}$. Elliptic hole the edge of which is subject to uniform tangential stress $T$.

$4^{\circ}$. Elliptic hole (or straight cut) part of the edge of which is subject to uniform pressure . . . . . .

$5^{\circ}$. Approximate solution of the problem of bending of a strip (beam) with an elliptic hole . . . . . .

$\S 83$. Solution of the second fundamental problem for the infinite plane with an elliptic hole . . . . . . . .

354

358

$\S$ 83a. Examples.

$1^{\circ}$. Uni-directional tension of an infinite plate with a rigid elliptic centre........... .

$2^{\circ}$. Case when the elliptic centre is not allowed to rotate 363

$3^{\circ}$. Case when a couple with given moment acts on the elliptic kernel . . . . . . . . . . . 366

$4^{\circ}$. Case when a force acts on the centre of the elliptic kernel .

$\S 84$. General solution of the fundamental problems for regions, mapped on to the circle by the help of polynomials 
$\S 85$. Generalization to the case of transformations by means of rational functions . . . . . . . . . . . 374

$\S 85$. Solution of the second fundamental problem. On the solution of the mixed fundamental problem . . . . 379

$\S 87$. Other methods of solution of the fundamental problems 379

$\S 87 a$. Example. Solution of the first fundamental problem for an infinite plane with a circular hole . . . . . .

$\S 88$. Further examples. Application to some other boundary

problems . . . . . . . . . . . . . . . . 384

$\S 89$. Application to the approximate solution of the general case

380

385

Chapter 16. Solution of the fundamental problems For the HALF-PLANE AND FOR SEMI-INFINITE REGIONS.

$\S$ 90. General formulae and propositions for the half-plane

$\S$ 91. The general formulae for semi-infinite regions . . . . 397

$\S$ 92. Basic formulae, connected with conformal transformation on to the half-plane . . . . . . . . . .

$\S$ 93. Solution of the first fundamental problem for the half-plane. . . . . . . . . . . . . . . 402

§ 93a. Example . . . . . . . . . . . . . . . . . . . . 406

$\S$ 94. Solution of the second fundamental problem . . . . 409

$\S 95$. Solution of the fundamental problems for regions, mapped on to the half-plane by means of rational functions. Case of a parabolic contour . . . . . . . 411

Chapter 17. Some general Methods of SOlution of Boundary value problems. Generalizations.

$\S$ 96. On the integral equations of S. G. Mikhlin . . . . . 414

$\S 97$. On a general method of solution of problems for multiply connected regions . . . . . . . . . . . 416

$\S 98$. The integral equations, proposed by the Author . . . 417

$\S$ 99. Application to contours with corners . . . . . . . 427

$\S 100$. On the numerical solution of the integral equations of the plane theory of elasticity. . . . . . . . . . . 427

$\S 101$. The integral equations of D. I. Sherman-G. Lauricella 427

$\S 102$. Solution of the first and second fundamental problems by the method of D. I. Sherman . . . . . . . . 
$\S 103$. On the solution of the mixed fundamental problem and of certain other boundary problems by means of $\mathrm{D}$. I. Sherman's method. . . . . . . . . . . . 440

$\S 104$. Generalization to anisotropic bodies . . . . . . . . 441

$\S 105$. On other applications of the general representation of solutions...... . . . . . . . . . . 441

PART VI - Solution of the boundary of the plane theory of elasticity by reduction to the problem of linear relationship . . . . . . 445

Chapter 18. The PROBLEM OF LINEAR Relationship.

$\S 106$. Sectionally holomorphic functions . . . . . . . . 447

$\S 107$. The problem of linear relationship (the Hilbert problem) 448

$\S 108$. Determination of a sectionally holomorphic function for a given discontinuity . . . . . . . . . . . . 449

$\S$ 109. Application . . . . . . . . . . . . . . 452

$\S$ 109a. Example . . . . . . . . . . . . . . 455

$\S 110$. Solution of the problem: $F^{+}=g F^{-}+f . . . .4456$

$\S 111$. Case of discontinuous coefficients . . . . . . 468

Chapter 19. Solution of the Fundamental PRoblems For the HALF-PLANE AND FOR THE PLANE WITH STRAIGHT CUTS.

$\S 112$. Transformation of the general formulae for the halfplane . . . . . . . . . . . . . . . . 471

$\S 113$. Solution of the first and second fundamental problems for the half-plane . . . . . . . . . . . . . 476

$\S 114$. Solution of the mixed fundamental problem . . . 478 $\S 114 \mathrm{a}$. Examples.

$1^{\circ}$. Stamp with straight horizontal base . . . . . 486

$2^{\circ}$. Stamp with straight inclined base . . . . . . . 488

$3^{\circ}$. Effect of asymmetrically distributed forces . . . 492

$\S 115$. The problem of pressure of rigid stamps in the absence of friction. . . . . . . . . . . . . 492

$\S 1$ 16. Application . . . . . . . . . . . . . 496

$\S 116 a$. Examples.

$1^{\circ}$. Stamp with straight horizontal base. . . . . 501

$2^{\circ}$. Stamp with straight inclined base...... . 501

$3^{\circ}$. Stamp with curved base . . . . . . . . 502 
$\S 117$. Equilibrium of a rigid stamp on the boundary of an elastic half-plane in the presence of friction . . . . . 504

$\S 117$ a. Examples.

$1^{\circ}$. Stamp with straight horizontal base. . . . . 508

$2^{\circ}$. Stamp with straight inclined base. . . . . . . 509

$\S 118$. An alternative method for the solution of the boundary problems for the half-plane. . . . . . . . . 510

$\S 119$. Problem of contact of two elastic bodies (generalized plane problem of Hertz) . . . . . . . . . . 510

$\S 120$. Boundary problems for the plane with straight cuts 515

Chapter 20. Solution of BOUndary problems For Regions, BOUNDED BY CIRCLES, AND FOR THE INFINITE PLANE, CUT ALONG CIRCULAR ARCS.

$\S 121$. Transformation of the general formulae for regions, bounded by a circle . . . . . . . . . . . . 525

$\S 122$. Solution of the first and second fundamental problems for the region, bounded by a circle . . . . . . . 529

$\S 123$. The mixed fundamental problem for a region, bounded by a circle . . . . . . . . . . . 531

$\S 123 a$. Example . . . . . . . . . . . . . . . 536

$\S 124$. Boundary problems for the plane, cut along circular arcs 538

$\S 124 a$. Example. Extension of the plane, cut along a circular arc 542

ChAPTER 21. SOLUTION OF THE BOUNDARY PROBLEMS FOR REGIONS, MAPPED ON TO THE CIRCLE BY RATIONAL FUNCTIONS.

$\S 125$. Transformation of the general formulae . . . . . 546

$\$ 126$. Solution of the first and second fundamental problems 552

$\S 127$. Solution of the mixed fundamental problem . . . . 554

$\S 127$ a. Example. Solution of the mixed fundamental problem for the plane with an elliptic hole . . . . . . . 558

$\S 128$. The problem of contact with a rigid stamp . . . . 560 $\S 128$ a. Examples.

$1^{\circ}$. Circular disc. . . . . . . . . . . 568

$2^{\circ}$. Infinite plane with a circular hole... . . 571

$3^{\circ}$. Infinite plane with an elliptic hole. . . . . . 574

PART VII - Extension, torsion and bending of homogeneous and compound bars. . . . . . . . . . . . . 579 
Chapter 22. Torsion AND Bending OF homogeneous bars (PROBlem of SAINT-Venant).

$\S 129$. Statement of the problem. . . . . . . . . 583

$\S 130$. Certain formulae. . . . . . . . . . . . . 586

$\S 131$. General solution of the torsion problem . . . . . 587

$\S 132$. Complex torsion function. Stress functions . . . . . 594

$\S 133$. On the solution of the torsion problem for certain particular cases . . . . . . . . . . . . . 597

$\S 134$. Application of conformal mapping. . . . . . . . . 599 $\S 134 \mathrm{a}$. Examples.

$1^{\circ}$. Epitrochoidal section . . . . . . . . . 602

$2^{\circ}$. Booth's lemniscate . . . . . . . . . . 604

$3^{\circ}$. The loop of Bernoulli's lemniscate . . . . . . 605

$4^{\circ}$. Confocal ellipses. Eccentric circles . . . . . . 607

$\S 135$. Extension by longitudinal forces . . . . . . . 607

$\S 136$. Bending by couples, applied to the ends . . . . . 608

$\S 137$. Bending by transverse forces . . . . . . . . . . 612

$\S 138$. On the solution of problems of bending for different cross-sections . . . . . . . . . . . . 618

$\S 138$ a. Example. Bending of a circular cylinder or tube . 619

Chapter 23. Torsion OF bars CONSISting of Different MATERIALS.

$\S 139$. General formulae . . . . . . . . . . . . . 621

$\S 140$. Solution by means of integral equations . . . . . 626

$\S 140$ a. Applications..

$1^{\circ}$. Torsion of a circular cylinder, reinforced by a longitudinal round bar of a different material . . . 630 $2^{\circ}$. Torsion of a rectangular bar, consisting of two different rectangular parts. . . . . . . 635

Chapter 24. Extension AND BENDING OF BARS, CONSISTING OF DIFFERENT MATERIALS WITH UNIFORM POISSON'S RATIO.

$\S 141$. Notation . . . . . . . . . . . . . . . . 640

$\S 142$. Extension. . . . . . . . . . . . . . 642

§143. Bending by a couple. . . . . . . . . . . . . . . 642

$\S 144$. Bending by a transverse force . . . . . . . . . . 643 
$\S 144 a$. Example. Bending of a compound circular tube by a transverse force, applied to one of its ends. . . . . 647

Chapter 25. Extension and Bending for different PoisSON'S RATIOS.

$\S 145$. An auxiliary problem of plane deformation . . . 650

$\S 146$. The problem of extension and of bending by a couple 652

$\S 147$. Particular cases.

$1^{\circ}$. Extension of a bar, having an axis of symmetry 662

$2^{\circ}$. Bar with plane of symmetry, bent by a couple . . 663

$\S 148$. Principal axis of extension and principal planes of bending. . . . . . . . . . . . . . . . 664

$\S 149$. Application of complex representation. Examples . . 670

$\S 150$. Problem of bending by a transverse force . . . . . 675

APPENDIX 1. On the concept of a tensor . . . . . . . . . 682

APPENDIX 2. On the determination of functions from their perfect differentials in multiply connected regions . . . . 697

APPENDIX 3. Determination of a function of a complex variable from its real part. Indefinite integrals of holomorphic functions . . . . . . . . . . . 708

AUTHORS INDEX AND REFERENCES . . . . . . . . . 713

SUPPLEMENTARY REFERENCES . . . . . . . . . . 727

SUBJECT INDEX . . . . . . . . . . . . . . . . . 729 